\title{
An Accurate Smartphone Battery Parameter Calibration Using Unscented Kalman Filter
}

\author{
Chalukya Bhat ${ }^{1}$, Student Member, IEEE, Aniruddh Herle ${ }^{2}$, Student Member, IEEE, Janamejaya Channegowda ${ }^{3}$, \\ Member, IEEE, Kali Naraharisetti ${ }^{4}$ Member, IEEE \\ ${ }^{1,2,3}$ M S Ramaiah Institute of Technology, Bangalore, India; ${ }^{4}$ Infineon Technologies \\ E-mail: chalukya.bhat@gmail.com ${ }^{1}$, aniruddh.herle@gmail.com ${ }^{2}$, bcjanmay.edu@gmail.com ${ }^{3}$, \\ swaraj.kali@gmail.com ${ }^{4}$
}

\begin{abstract}
Internet of Things (IoT) applications have opened up numerous possibilities to improve our lives. Most of the remote devices, part of the IoT network, such as smartphones, data loggers and wireless sensors are battery powered. It is vital to collect battery measurement data (Voltage or State-ofCharge (SOC)) from these remote devices. Presence of noise in these measurements restricts effective utilization of this dataset. This paper presents the application of Unscented Kalman Filter (UKF) to mitigate measurement noise in smartphone dataset. The simplicity of this technique makes it a constructive approach for noise removal. The datasets obtained after noise removal could be used to improve data-driven time series forecasting models which aid to accurately estimate critical battery parameters such as SOC. UKF was tested on noisy charge and discharge dataset of a smartphone. An overall Mean Squared Error (MSE) of 0.0017 and 0.0010 was obtained for Voltage charge and discharge measurements. MSE for SOC charge and discharge data measurements were 0.0018 and 0.0010 respectively.
\end{abstract}

Keywords: Unscented kalman filter, Battery, Energy Storage, Measurement, Denoise

\section{INTRODUCTION}

Increase in number of IoT enabled devices have led to significant improvements in home automation [1]. Remote devices are primarily powered by Lithium-ion based batteries. Advancements in energy storage systems have not kept pace with growth of IoT devices [2]. Smartphones, one of the most commonly available IoT device, over the past decade have become a ubiquitous part of our daily lives. Unanticipated smartphone shutdown have hampered the device usage. Accurate State-of-Charge (SOC) estimation aids to predict the available usage time of a smartphone [3].

SOC estimation of batteries is challenging due to the inherent non-linear battery characteristics. Added to this nonlinearity, batteries suffer from capacity fade, the collective capacity of the battery reduces overtime, this reduction is illustrated in Fig 1. Public battery datasets are available [4], [5], but are collected in highly controlled environments. Collection of smartphone battery data is needed to evaluate and anticipate the approximate time-limit for device shutdown.

Presence of noise in such datasets is a cause for concern as they negatively impact the inference obtained from datadriven models [6] - [8]. Data cleaning and curation is a vital pre-processing task in all data-driven techniques [9].

There have been multiple research endeavors to use UKF for
SOC estimation [10] - [12]. This paper is the first to address the issue of smartphone battery noise removal using UKF. For the sake of completeness the equivalent circuit model used is illustrated in Fig 2 [13]. Section II of this paper provides details about UKF followed by discussion of results in Section III. Conclusion of the present work is presented in Section IV.

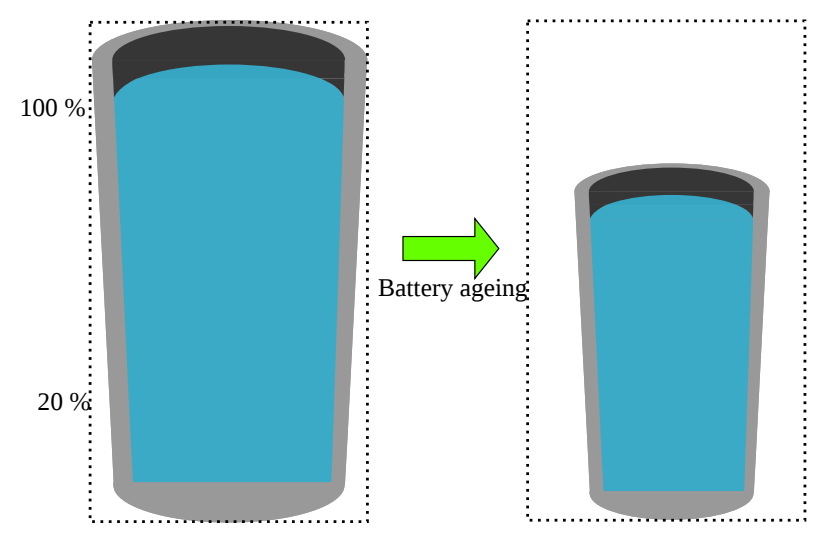

Fig. 1: Battery capacity fade overtime

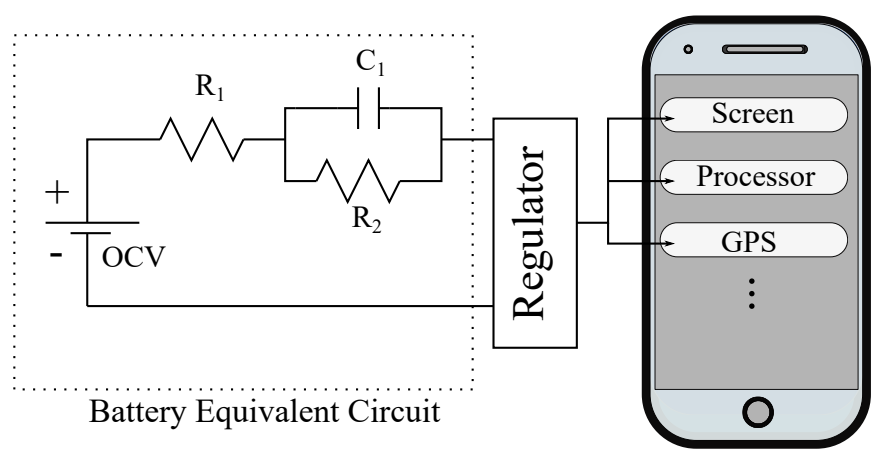

Fig. 2: Smartphone Battery Equivalent Circuit

\section{UNSCENTED KALMAN FILTER}

Unscented kalman filter employs a nonlinear unscented transformation (UT), UKF performs estimation by iterative update in the mean and error covariance. UKF is advantageous for estimating the properties of a random variable subjected to nonlinear transformation. UKF is helpful for noise reduction in the state variables of non-linear functions by adopting initial 
values signal under consideration and evaluating future values iteratively. The State and Measurement Equations are provided below:

$$
\begin{aligned}
& \mathbf{x}_{k}=\mathbf{f}\left(\mathbf{x}_{k-1}\right)+\mathbf{w}_{k-1} \\
& \mathbf{z}_{k}=\mathbf{h}\left(\mathbf{x}_{k}\right)+\mathbf{v}_{k}
\end{aligned}
$$

In the equation $\mathbf{x}_{(k-1)}$ is the state vector at the moment $k-1$ and $\mathbf{x}_{k}$ is the state vector at the instance $k . \mathbf{w}_{k-1}$ is the process noise at $k-1$ and $\mathbf{v}_{k}$ is the measurement noise at $k . \mathbf{f}\left(\mathbf{x}_{k-1}\right)$ and $\mathbf{h}\left(\mathbf{x}_{k}\right)$ are non-linear functions [14] - [16]. The procedure followed for noise removal is given below:

Step 1:The State equations of the terminal Voltage $E_{T}$ and state-of-charge $S O C$ are given as here:

$$
\begin{gathered}
E_{T(t+1)}=E_{T(t)}+\frac{V_{1}}{R_{1}\left(S O C, T_{b}\right) * C_{1}\left(S O C, T_{b}\right)}- \\
\frac{I}{C_{1}\left(S O C, T_{b}\right)}+V_{t} \\
S O C_{T+1}=\mathrm{SOC}_{T}+-\frac{I}{3600 \cdot C_{q}} T_{s}+V_{t}
\end{gathered}
$$

Where $V_{t}$ is the process noise, $T_{s}$ is time step which is set to 1 second and $C_{q}$ is the capacity of the smartphone battery. The Measurement equations used to determine clean terminal voltage and state of charge are:

$$
\begin{gathered}
\widehat{E_{T(t)}}=E_{T(t)}+W_{t} \\
S \widehat{O C}_{(t)}=S O C_{(t)}+W_{t}
\end{gathered}
$$

The symbols used have been defined below:

- $W_{t}=$ Measurement Noise

- $R_{1}=$ Equivalent battery resistance

- $V_{1}=$ Voltage across $R$ and $C$ as seen in Fig 3

- $V_{1}$ and $S O C$ are preliminary values

- Current $I$ and battery temperature $T_{b}$ are obtained from logged data

Step 2: White noise of value $10^{-4} \frac{W}{H z}$ is added to the measured value of terminal voltage $\left(E_{T}\right)$.

Step 3: UKF parameters including the initial covariences are defined as provided in Table III

Step 4: Compare UKF output and with the dataset and calculate Mean Square Error (MSE). The block diagram of the approach is highlighted in Fig 8

\section{A. Battery Equivalent Circuit}

Battery Equivalent Circuit model is provided in Fig 3 Passive Battery parameters $R_{1}$ and $C_{1}$ are a function of State of Charge and battery and temperature. To improve predictions of UKF thermal effects of the battery are also considered. The resistance and capacitance values at temperatures $278 \mathrm{~K}, 293 \mathrm{~K}$ and $313 \mathrm{~K}$ are provided in table $\mathrm{I}$ and table $\mathrm{II}[13]$.

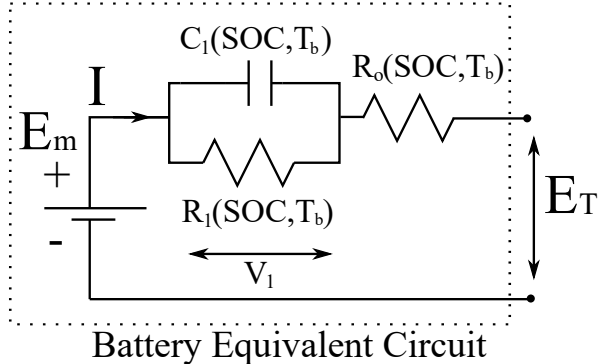

Fig. 3: Battery Equivalent Circuit illustrating passive elements

TABLE I: Table Providing resistance $R_{1}$ values at temperatures $278 \mathrm{~K}, 293 \mathrm{~K}$ and $313 \mathrm{~K}$ for variations in SOC

\begin{tabular}{lccc}
\hline SOC & $R_{278 K} \Omega$ & $R_{293 K} \Omega$ & $R_{313 K} \Omega$ \\
\hline 0 & 0.0109 & 0.0029 & 0.0013 \\
0.1 & 0.0069 & 0.0024 & 0.0012 \\
0.25 & 0.0047 & 0.0026 & 0.0013 \\
0.5 & 0.0034 & 0.0016 & 0.001 \\
0.75 & 0.0033 & 0.0023 & 0.0014 \\
0.9 & 0.0033 & 0.0018 & 0.0011 \\
1 & 0.0028 & 0.0017 & 0.0011 \\
\hline
\end{tabular}

TABLE II: Table Providing Capacitance $C_{1}$ values at temperatures $278 \mathrm{~K}, 293 \mathrm{~K}$ and $313 \mathrm{~K}$ for variations in SOC

\begin{tabular}{lccc}
\hline SOC & $C_{278 K \mu F} \mu$ & $C_{293 K \mu F}$ & $C_{313 K} \mu F$ \\
\hline 0 & 1913.6 & 12447 & 30609 \\
0.1 & 4625.7 & 18872 & 32995 \\
0.25 & 23306 & 40764 & 47535 \\
0.5 & 10736 & 18721 & 26325 \\
0.75 & 18036 & 33630 & 48274 \\
0.9 & 12251 & 18360 & 26839 \\
1 & 9022.9 & 23394 & 30606 \\
\hline
\end{tabular}

\section{RESULTS AND DISCUSSION}

All measurements with an added White Noise of value $10^{-4}$ $\frac{W}{H z}$ are shown in Fig 4, Fig 5 , Fig 6 and Fig 7 respectively. The block diagram displaying noise addition is in Fig 8 Preliminary error rate was assumed to be $10 \%$ of terminal voltage. All the other smartphone battery parameters are taken from the datasheet [17]. The filtered output voltage has been compared with ground truth values as shown in Fig 9

TABLE III: UKF parameters

\begin{tabular}{lc}
\hline Parameter & Value \\
\hline Process Noise Covariance & 0.5 \\
Initial State Voltage (charge) & $2.5 \mathrm{~V}$ \\
Initial State Voltage (discharge) & $4.2 \mathrm{~V}$ \\
Initial state of charge (charge) & 0.01 \\
Initial state of charge (discharge) & 1 \\
Initial state Covariance (voltage) & 0.01 \\
Initial state Covariance (SOC) & 0.01 \\
Measurement Noise Covariance & 1 \\
Battery Capacity $\left(C_{q}\right)$ & $4000 \mathrm{mAh}$ \\
\hline
\end{tabular}

\section{A. Mean Squared Error}

To assess the standard of UKF output, Mean Squared Error (MSE) was used. MSE provides the average squared difference between the estimated and the ground truth voltage value. The 


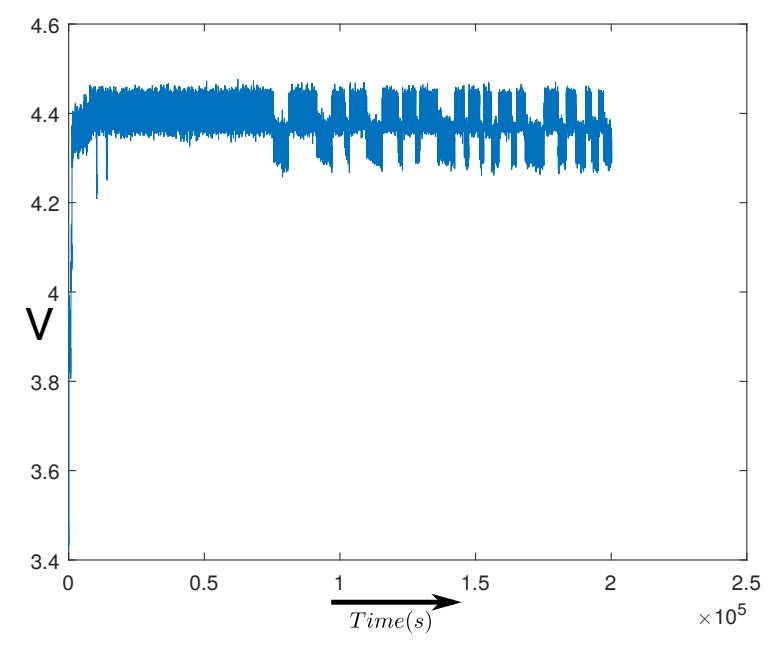

Fig. 4: Presence of noise in battery voltage during charging

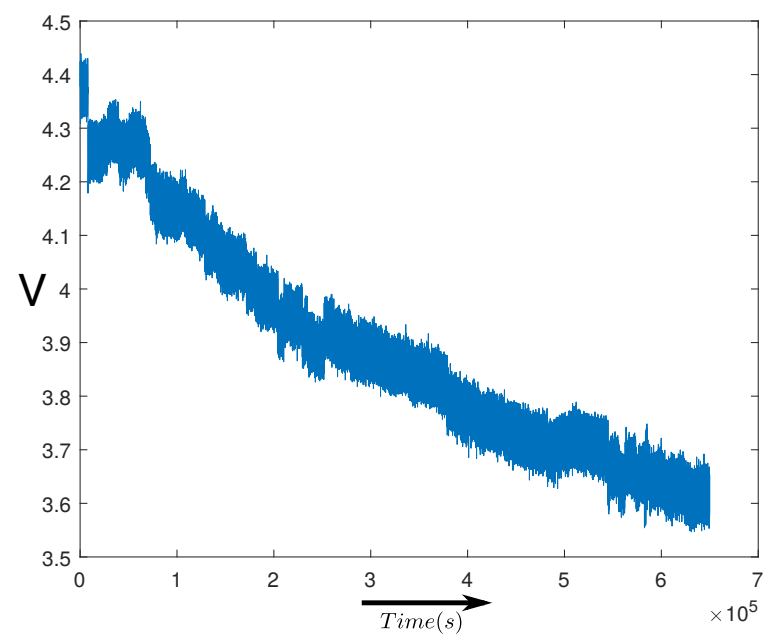

Fig. 5: Presence of noise in battery voltage during discharge

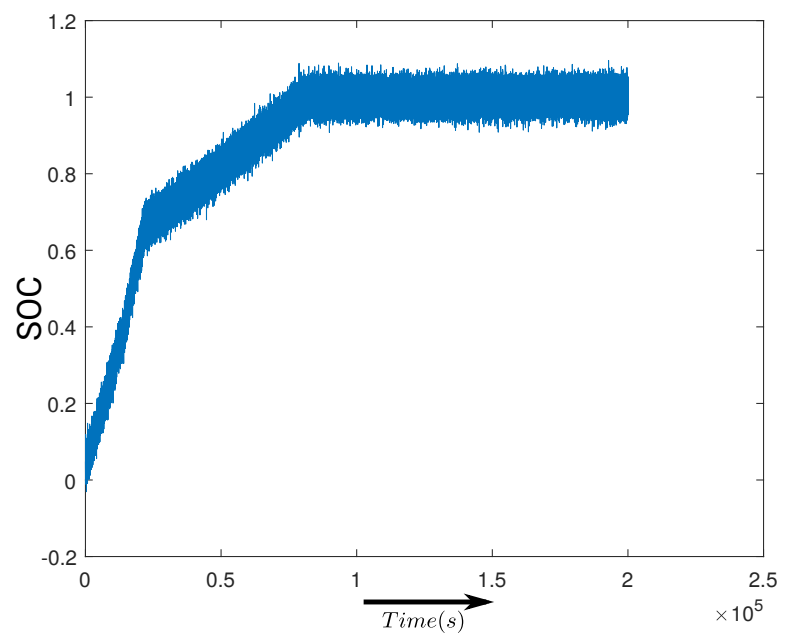

Fig. 6: Presence of noise in battery SOC during charging

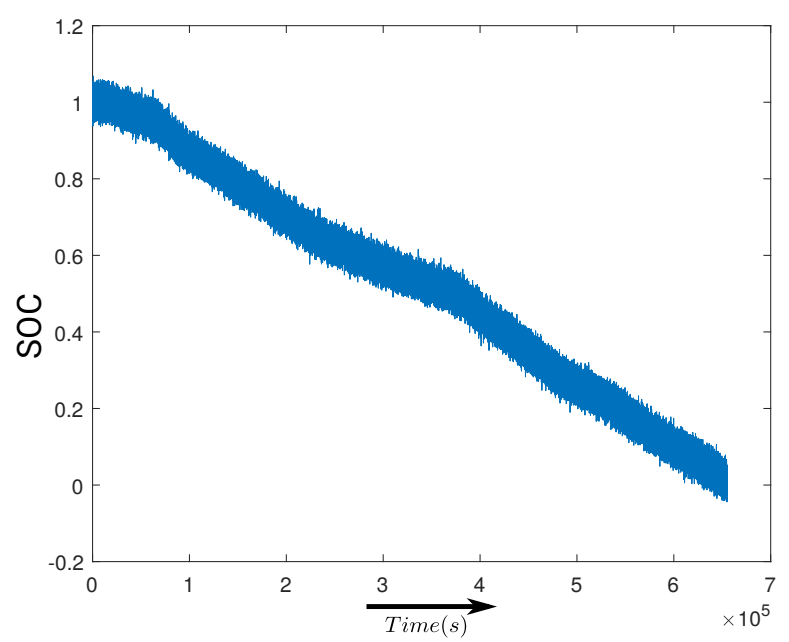

Fig. 7: Presence of noise in battery SOC during discharge

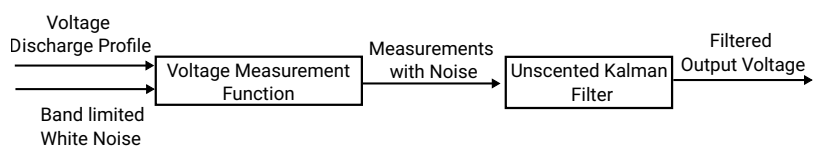

Fig. 8: Block Diagram of UKF

number of data points selected for this study was 25000 and 70000 for charging and discharging respectively.

$$
M S E=\frac{1}{n} \sum_{i=1}^{n}\left(Y_{i}-\hat{Y}_{i}\right)^{2}
$$

- MSE = Mean Squared Error

- $\mathrm{n}=$ number of data points

- $Y_{i}=$ Observed Values

- $\hat{Y}_{i}=$ Predicted Values

\section{CONCLUSION}

Removal of noise in measured data is important to extract maximum value from the dataset. This paper explored the use of Unscented Kalman Filter (UKF) to mitigate noise in measured data. The voltage \& State-of-Charge measurements were selected to test the proposed approach. This technique does not need extensive computational capabilities and can be deployed in most smartphones with ease. UKF resulted in a Mean Squared Error (MSE) of 0.0017 and 0.0010 for charge and discharge measurements of smartphone battery voltage. A MSE of 0.0018 and 0.0010 was obtained for SOC measurements for charge \& discharge conditions. 


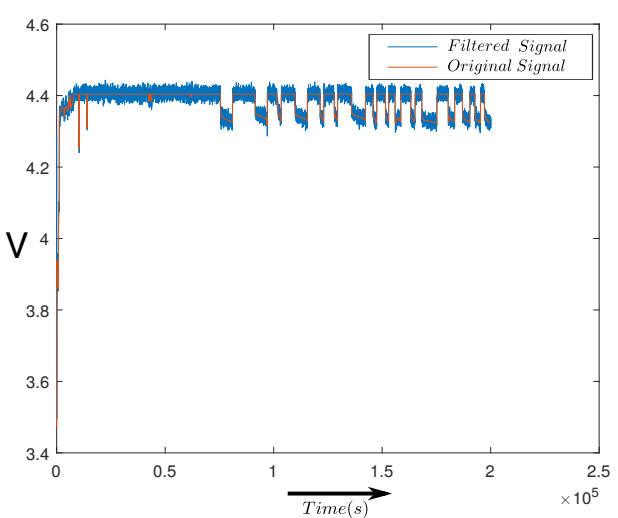

(a)

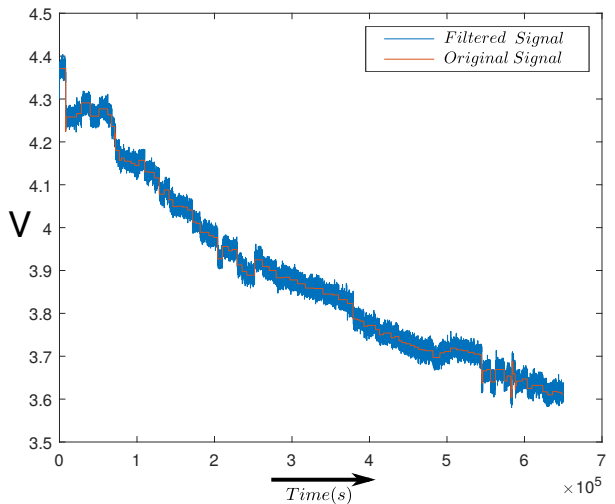

(c)
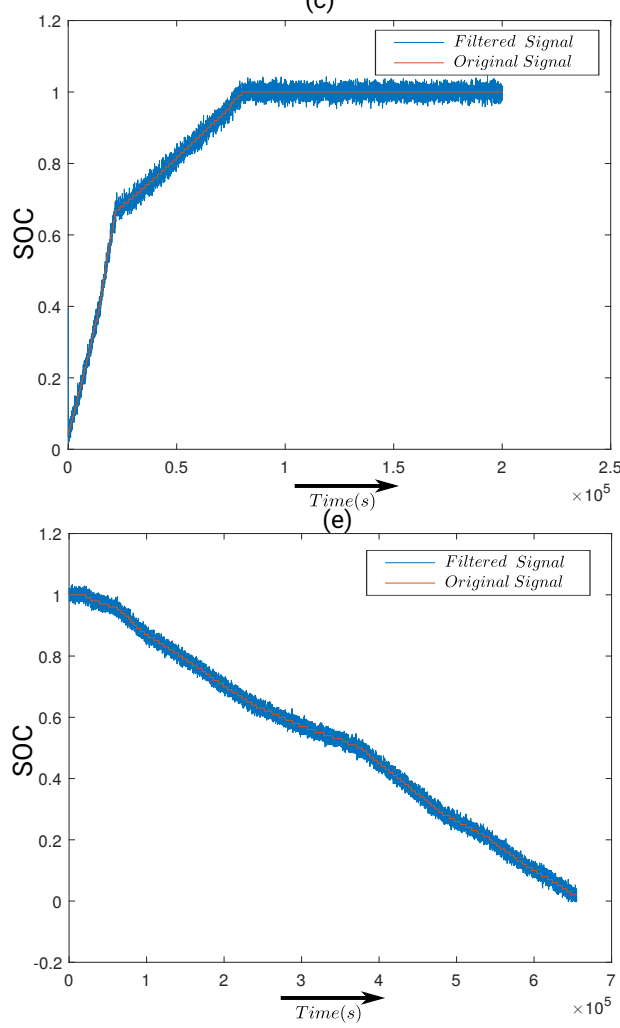

(g)

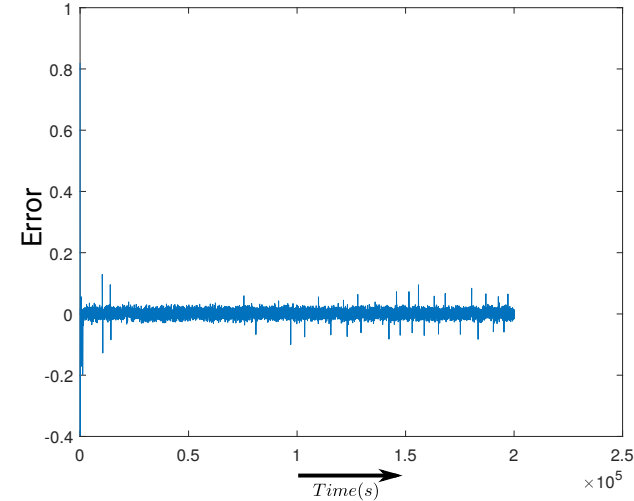

(b)

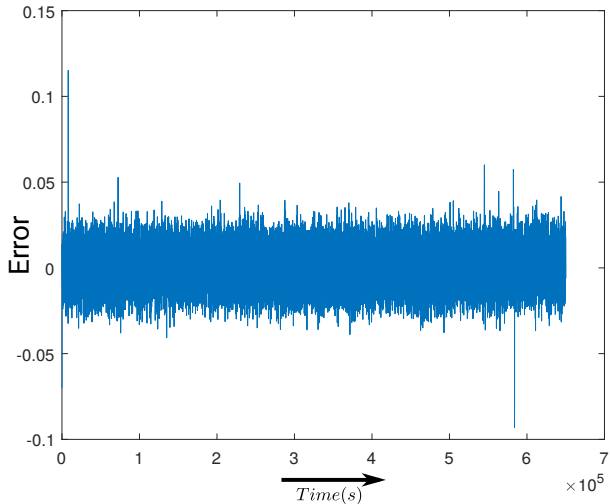

(d)
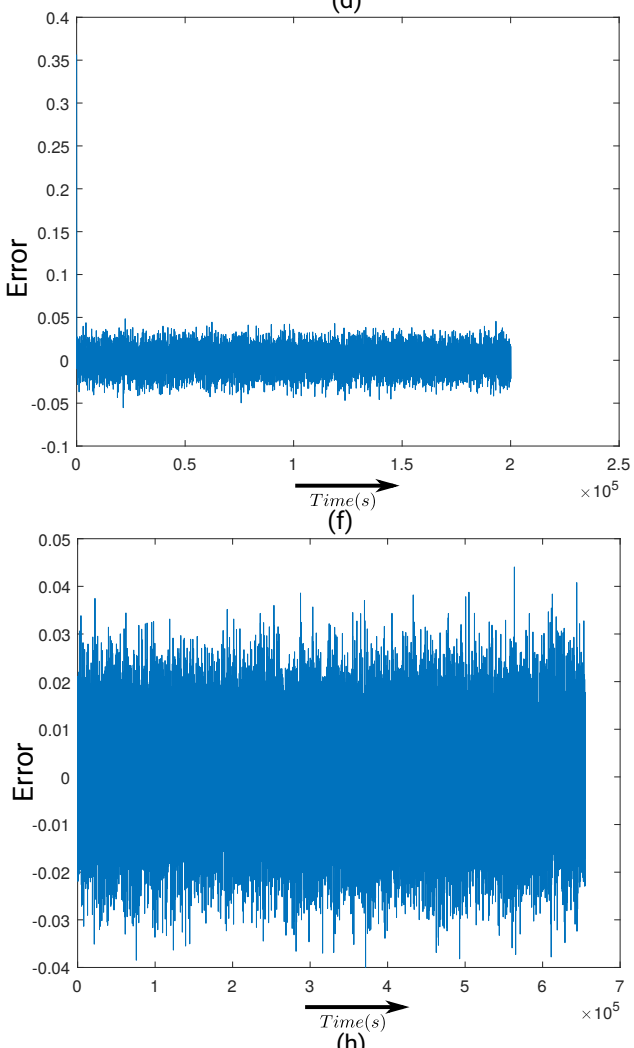

(h)

Fig. 9: Output of UKF for charging voltage characteristics is shown in (a) the error is shown in (b). The discharging characteristics of the battery are compared with ground truth values in (c) and the error is illustrated in (d). Ground truth values for State-of-charge (SOC) for charging and discharging is shown in (e) and (g). Error plots for SOC are depicted in (f) and (h) respectively. Total simulation time was 2500 seconds with a simulation time step of 0.1 second for battery charging dataset \& 7000 seconds with a 0.1 second during discharging 


\section{REFERENCES}

[1] Satyendra K. Vishwakarma, Prashant Upadhyaya, Babita Kumari and Arun Kumar Mishra, "Smart Energy Efficient Home Automation System Using IoT", 4th International Conference on Internet of Things: Smart Innovation and Usages (IoT-SIU), July, 2019.

[2] A. Adhikaree, T. Kim, J. Vagdoda, A. Ochoa, P. J. Hernandez and Y. Lee, "Cloud-based battery condition monitoring platform for largescale lithium-ion battery energy storage systems using Internet-of-Things (IoT)", Proc. 2017 IEEE Energy Conversion Congress and Exposition, pp. 1004-1009, Oct. 1-5, 2017

[3] R. Xiong, Y. Zhang, J. Wang, H. He, S. Peng, and M. Pecht, "Lithiumion battery health prognosis based on a real battery management system used in electric vehicles," IEEE Trans. Veh. Technol., to be published

[4] B. Saha and K. Goebel (2007). "Battery Data Set", NASA Ames Prognostics Data Repository (http://ti.arc.nasa.gov/project/prognostic-datarepository), NASA Ames Research Center, Moffett Field, CA

[5] Kollmeyer, Phillip (2018), "Panasonic 18650PF Li-ion Battery Data", Mendeley Data, V1, doi: 10.17632/wykht8y7tg.1

[6] T. J. Witt and Yi-hua Tang, "Investigations of noise in measurements of electronic voltage standards," in IEEE Trans. Instrum. Meas., vol. 54, no. 2, pp. 567-570, April 2005.

[7] C. Wang, X. Wei and P. Zhou, "Optimize Scheduling of Federated Learning on Battery-powered Mobile Devices," 2020 IEEE International Parallel and Distributed Processing Symposium (IPDPS), New Orleans, LA, USA, 2020, pp. 212-221.

[8] H. Wang, S. Sievert, S. Liu, Z. Charles, D. Papailiopoulos and S. Wright, "ATOMO: Communication-efficient learning via atomic sparsification", Proc. Advances in Neural Information Processing Systems, pp. 1-12, 2018.
[9] V. Kumar and C. Khosla, "Data Cleaning - A thorough analysis and survey on unstructured data", 2018 8th International Conference on Cloud Computing Data Science \& Engineering (Confluence), pp. 5, 2018.

[10] V. Sangwan, R. Kumar and A. K. Rathore, "State-of-charge estimation for li-ion battery using extended Kalman filter (EKF) and central difference Kalman filter (CDKF)", 2017 IEEE Industry Applications Society Annual Meeting, pp. 1-6, 2017

[11] L. Haoran, L. Liangdong, Z. Xiaoyin and S. Mingxuan, "Lithium Battery SOC Estimation Based on Extended Kalman Filtering Algorithm”, 2018 IEEE 4th International Conference on Control Science and Systems Engineering (ICCSSE), pp. 231-235, 2018.

[12] P. A. Topan, M. N. Ramadan, G. Fathoni, A. I. Cahyadi and O. Wahyunggoro, "State of charge (SOC) and state of health $(\mathrm{SOH})$ es timation on lithium polymer battery via Kalman filter", Proc. 2nd Int. Conf. Sci. Technol.-Comput. (ICST), pp. 93-96, Oct. 2016.

[13] T. Huria, M. Ceraolo, J. Gazzarri and R. Jackey, "High fidelity electrical model with thermal dependence for characterization and simulation of high power lithium battery cells", Proc. 2012 IEEE Int. Electric Vehicle Conference (lEVC), pp. 1-8.

[14] G. A. Terejanu, Unscented Kalman Filter Tutorial, July 2006.

[15] E. A. Wan and R. van der Merwe, "The Unscented Kalman Filter for Nonlinear Estimation”, Proc. of IEEE Symposium 2000 (AS-SPCC), Oct. 2000

[16] I. Jokic, Z. Zecevic and B. Krstajic, "State-of-charge estimation of lithium-ion batteries using extended Kalman filter and unscented Kalman filter", Proc. 23rd Int. Sci.-Prof. Conf. Inf. Technol. (IT), pp. 1-4, Feb. 2018.

[17] Battery Chargers and Charging Methods. https://www.mpoweruk.com/ chargers.htm 